\title{
Application of Finite Elastic Theory to the Deformation of Rubbery Materials*
}

PALI. J. BLATZ and WILLIAM L. KO, Guggenheim Aeronautical Laboratory, California Institute of Technology, Pasadena, California

\section{Introduction}

Since Hooke ${ }^{1}$ enunciated his famous "ut tensio sic vis" in 1678 , there have been many attempts by theoreticians to formulate more general relations between stress and strain to account for behavior at large deformation. As a result of the formalism of continuum elasticity enunciated by Rivlin, ${ }^{2}$ Reiner. ${ }^{3}$ and Truesdell, ${ }^{4}$ there has developed a rational foundation for the analytical representation of the elastic deformation of continuous bodies. Inherent in these representations is the notion of a strain energy function $W$, which, for continuous isotropic media, is a function only of certain invariant properties, which in turn are proper to the deformation tensor. Beyond this, little more can be said about the nature of this function $W$. It is true that, when represented as a power series expansion in its invariants, certain identifications of the leading terms with infinitesimal Hooke-Cauchy theory can be established. It. is further established experimentally that it is difficult from experiments on brittle plastics or metals to define accurately coefficients beyond the leading terms. It follows that rubbery materials, many of which evince up to $700 \%$ ultimate extension ratio, are ideal for this purpose.

The purpose of this discussion, then, is to show how the nature of the strain energy function can be deduced from experiments on rubbery materials. A great deal of work ${ }^{5}$ has already been done along this line. Most of this work is, however, limited to nearly incompressible materials, and in the course of the data reduction, incompressibility was assumed. It is our intent not to assume incompress-

* This research was supported in part by a subcontract from the Polaris division of the Aerojet General Corporation, Sacramento, California. 
ibility, and, thereby, to show what form the dilatation takes in large strain theory.

In order to have a highly dilatable elastic material, a batch of polyurethane foam rubber was prepared by leaching out salt from a filled composite. The resultant foams have approximately $50 \%$ vol. voids, about $40 \mu$ in diameter. This material served the purpose very well, as will be seen later, and in addition, was mechanically ideal in that no hysteresis was observed in any of the data obtained in each of three different stress fields. A sequence of studies similar to the one here reported is now under way on foams of void content ranging from 0 to $90 \%$. It is to be anticipated that the mechanical behavior of the highly voided foams will be much more complex than that reported herewith.

A second but somewhat premature conclusion drawn from these studies is a geometrical evaluation of the failure criterion. This is presented primarily to indicate the possible ways that one can plot failure surfaces for materials which fracture at large strains.

\section{The Constitutive Law in Finite Strain Theory}

\section{A. General Stress Field}

Consider a deformation in which a point of an isotropic elastic body initially having Cartesian coordinates $P_{\mathrm{u}}\left(x^{i}\right)$ is displaced to a new Cartesian position $P\left(X^{i}\right)$. The deformation tensor which characterizes this mapping is denoted by:

$$
G_{i k}=\frac{\partial X^{m}}{\partial X^{i}} \frac{\partial X^{m}}{\partial x^{k}}
$$

and the physical stresses resulting in the body after this deformation ${ }^{6}$ are given by:

$$
\sigma_{\mathfrak{i} k}=\frac{2}{\sqrt{I_{3}}}\left[\frac{\partial W}{\partial I_{1}} G_{i k}-I_{3} \frac{\partial W}{\partial I_{2}}\left(G^{-1}\right)_{i k}+\left(I_{2} \frac{\partial W}{\partial I_{2}}+I_{3} \frac{\partial W}{\partial I_{3}}\right) \delta_{i k}\right]
$$

The funetions $I_{1}, I_{2}$, and $I_{3}$ are the invariants of the deformation tensor, and are given by :

$$
\begin{gathered}
I_{1}=G_{k k} \\
I_{?}=1 / 2\left(I_{1}^{2}-G_{i k} G_{k i}\right)
\end{gathered}
$$




$$
I_{3}=\left|G_{i k}\right|
$$

The strain energy function $W$, measured per unit volume of undeformed body, is a function only of these invariants in the case of an isotropic material. It is our purpose to evaluate the gradients of $W$ with respect to these invariants.

It is convenient to introduce a new set of invariants.

$$
\begin{gathered}
J_{1}=I_{1}=G_{k k} \\
J_{2}=I_{2} / I_{3}=\left(G^{-1}\right)_{k k} \\
J_{3}=\sqrt{I_{\mathrm{a}}}=\tau / \tau_{0}=\sqrt{\left|G_{1 k}\right|}
\end{gathered}
$$

The invariant (8) is the ratio of the volume of an element of the deformed body to that of the undeformed body. After substitution, (2) becomes

$$
\begin{gathered}
\sigma_{i k}=\frac{2}{J_{3}}\left[W_{1} G_{i k}-W_{2}\left(G^{-1}\right)_{i k}\right]+W_{3} \delta_{i k}, \text { where } \\
W_{k}=\partial W / \partial J_{k}
\end{gathered}
$$

Consider now the special case of a uniform orthogonal deformation field, i.e.- the deformation tensor has only diagonal components $\lambda_{1}{ }^{2}, \lambda_{2}{ }^{2}, \lambda_{3}{ }^{3}$, where

$$
\lambda_{i}=\partial X_{i} / \partial x_{i}
$$

In this case, (9) becomes:

$$
\bar{\sigma}_{i} J_{3}=\sigma_{3} \lambda_{i}=2\left[W_{1} \lambda_{i}{ }^{2}-\frac{W_{2}}{\lambda_{i}{ }^{2}}\right]+J_{3} W_{3} \quad(i \text { not summed })
$$

and (6)-(8) become:

$$
\begin{gathered}
J_{1}=\Sigma \lambda_{i}^{2} \\
J_{2}=\Sigma \lambda_{i}{ }^{2} \\
J_{3}=\Pi \lambda_{i}
\end{gathered}
$$

where $\bar{\sigma}_{i}$ is the true stress on the deformed cross section, and $\sigma_{i}$ is the so-called engineering stress on the undeformed cross section.

Before proceeding to apply (12) to experimental data, let us investigate-the small strain behavior of $\left\{W_{1} W_{2} W_{3}\right\}$ in order to establish the 
character of the leading terms. The strain energy may be expanded as a power series in its invariants:

$$
W=\Sigma C_{l m n}\left(J_{1}-3\right)^{l}\left(J_{2}-3\right)^{m}\left(J_{3}-1\right)^{n}
$$

where $C_{000}=0$, since the reference state is undeformed. The deformation invariants can be expressed in terms of the small strain invariants as follows:

$$
\lambda_{i}=1+e_{i}
$$

where $e_{i}=\partial u_{i} / \partial x_{i}$ is the so-cilled Hookean strain.

$$
\begin{gathered}
J_{1}=3+2 \vartheta+\vartheta^{2}-2 \vartheta_{2} \approx 3+2 \vartheta \\
J_{2}=3-2 \vartheta+3 \vartheta^{2}-6 \vartheta_{1} \approx 3-2 \vartheta \\
J_{3}=1+\vartheta+\vartheta_{2}+\vartheta_{3} \approx 1+\vartheta
\end{gathered}
$$

where

$$
\begin{gathered}
\vartheta=\Sigma e_{i} \\
\vartheta_{2}=\sum_{i \neq j} e_{i} e_{j} \\
\vartheta_{3}=\Pi e_{i}
\end{gathered}
$$

After differentiating $(16)$ with respect to the $J$-invariants, substituting the $\vartheta$-variants, and grouping terms, the $W$-gradients are given by:

$$
\begin{aligned}
& W_{1}=A+B \vartheta+\cdots \\
& W_{2}=C+D \vartheta+\cdots \\
& W_{3}=E+F \vartheta+\cdots
\end{aligned}
$$

The second half of e(1. (12) can now be rewritten, up to linear terms, as:

$$
\begin{aligned}
\sigma_{i} \approx\left(1-e_{i}\right) & {\left[2\left(1+2 e_{i}\right)(A+B \vartheta)\right.} \\
- & \left.-2\left(1-2 e_{i}\right)(C+D \vartheta)+(1+\vartheta)(E+F \vartheta)\right]
\end{aligned}
$$

which is to be compared with Hooke's Law:

$$
\sigma_{i}=2 \mu e_{i}+(K-2 \mu / 3) \vartheta
$$

This leads immediately to three relations among the six parameters $(A \sim F)$ : 


$$
\begin{gathered}
2 A-2 C+E=0 \\
2 A+6 C-E=2 \mu \\
2 B+2 D+E+F=K-2 \mu / 3
\end{gathered}
$$

from which results

$$
A+C=\mu, 2
$$

It is observed that the parameters $A$ and $C$ are closely related to the Mooney-Rivlin parameters $C_{1}$ and $C_{2}$. (In fact, $C_{1}=A$, and $C_{2}$ $=C / J_{3}{ }^{2}$, so that constant $C$ does not imply constant $C_{2}$, and vice versa.) After introducing the notation:

$$
\begin{gathered}
A=\mu f / 2 \\
C=\mu(1-f) / 2
\end{gathered}
$$

there results:

$$
\begin{gathered}
E=\mu(1-2 f) \\
2 B-2 D+F=K-\mu(5 / 3-2 f)
\end{gathered}
$$

We shall be interested in a further specialization to materials which evince a behavior such that $W_{1}$ and $W_{2}$ are constant, i.e. $-B, D$, and the coefficients of higher terms in (24) and (25) are zero. For these materials:

$$
F=K-\mu(3 / 3-2 f)
$$

In addition, since $W_{1}$ and $W_{2}$ were constants, $W_{13}$ and $W_{23}$ are zero; and, therefore, $W_{3}$ is independent of $J_{1}$ and $J_{2}$. In view of (20), (35), and (37), (26) becomes:

$$
\begin{aligned}
& W_{3}=\mu(1-2 f)+[K-\mu(3 / 3-2 f)]\left(J_{3}-1\right) \\
&+ {\left[\ldots \text { higher powers of }\left(J_{3}-1\right) \ldots\right] }
\end{aligned}
$$

The constitutive relation hecomes:

$$
\bar{\sigma}_{i} J_{3}=\sigma_{i} \lambda_{i}=\mu\left[f \lambda_{i}{ }^{2}-\frac{1-f}{\lambda_{i}{ }^{2}}\right]+J_{3} W_{3}\left(J_{3} \text { only }\right)
$$

Since the principal stress difference is independent of $W_{3}$, the use of it permits one to determine $\{\mu, f\}$ directly by plotting: 


$$
\begin{gathered}
\frac{\sigma_{i} \lambda_{i}-\sigma_{j} \lambda_{j}}{\lambda_{i}{ }^{2}-\lambda_{j}{ }^{2}}=\mu\left[f+\frac{1-f}{\lambda_{i}{ }^{2} \lambda_{j}{ }^{2}}\right] \text { vs. } \frac{1}{\lambda_{i}{ }^{2} \lambda_{j}{ }^{2}} \\
\text { B. Specific Strese Fields }
\end{gathered}
$$

In order to determine $W_{3}$, it is necessury to express the $\lambda_{t}$ as functions of $J_{3}$ and to evaluate (39) for cases in which the left-hand side is zero.

(1) Simple Tension: For this case,

$$
\begin{gathered}
\lambda_{1}=\lambda, \lambda_{2}=\lambda_{3}=\lambda_{\mathrm{ltt}} ; \sigma_{1}=\sigma_{\mathrm{uni}}, \sigma_{2}=\sigma_{3}=0 \\
J_{3}=\lambda \lambda_{\mathrm{lat}^{2}}{ }^{2} \\
\frac{\lambda \sigma_{\mathrm{uni}}}{\lambda^{2}-\lambda_{\mathrm{lat}}{ }^{2}}=\mu\left[f+\frac{1-f}{\lambda^{2} \lambda_{\mathrm{lat}}{ }^{2}}\right] \\
0=\mu\left[f \lambda_{\mathrm{lat}}{ }^{2}-\frac{1-f}{\lambda_{\mathrm{lat}}{ }^{2}}\right]+J_{3} W_{3}
\end{gathered}
$$

Note that (43) is obtained from (40) by setting $i=1$, and $j=2$ or 3 ; while (44) is obtained from (39) by setting $j=2$ or 3 . Using (42) it follows that

$$
W_{3}=-\mu\left[\frac{f}{\lambda}-\frac{(1-f) \lambda}{J_{3}^{2}}\right]
$$

It remains to relate $J_{3}$ and $\lambda$. To this cnd, we introduce an ad hoc assumption, which turns out to correlate the data very nicely. We set:

$$
J_{3}=\lambda^{1-2 \nu}
$$

or equivalently

$$
\lambda_{\text {lat }}=\lambda^{-\nu}
$$

For small strains, (47) may be linearized to

$$
\epsilon_{\mathrm{lat}}=-\nu \epsilon
$$

showing that the parameter has the usual significance of Poisson's ratio. It is to be emphasized that the relation (46) is not unique to finite elastic theory. There are many ways in which the dilatation can be expressed in terms of a parameter related to Poisson's ratio of linear theory. Which of these functions is useful can be decided only 
by experimental evidence. Once having decided, however, that a given function fits the data bettter than others, it then behooves us to investigate the physical nature of rubbers further to see if the particular function can be derived from molecular statistics. We shall pursue this point later.

Now using (40), (44) becomes

$$
J_{3} W_{3}=-\mu\left[f J_{3}^{-2 \nu /(1-2 v)}-(1-f) J_{3}{ }^{2 \nu /(1-2 \nu)}\right]
$$

which, after integration and use of (33), (34) leads to

$$
\begin{aligned}
W=\mu f / 2\left[J_{1}-3+\frac{1-2 \nu}{\nu}\{\right. & \left.\left.J_{3}{ }^{-2 \nu /(1-2 \nu)}-1\right\}\right]+\mu(1-f) / 2\left[J_{2}\right. \\
& \left.-3+\frac{1-2 \nu}{\nu}\left\{J_{3}{ }^{2 \nu /(1-2 v)}-1\right\}\right]
\end{aligned}
$$

The value of eq. (50), which is an isothermal elastic equation of state for large strains, lies in its ability to predict stress-deformation behavior in any stress or displacement field. As we shall see, it does very nicely for foam rubbers. In order to apply it to continuum rubbers, however, very precise experimental large strain data in certain stress fields (close to hydrostatic) are needed to evaluate the dilatation terms

$$
J_{3} \pm 2 v /(1-2 v)
$$

since it is known that linear $\nu$ is 0.49997 , and, therefore, that the exponent is of the order of $=3 \times 10^{4}$.

The constitutive law associated with (50) is:

$$
\begin{array}{r}
\tilde{\sigma}_{i} J_{3}=\sigma_{i} \lambda_{i}=\mu f\left[\lambda_{i}{ }^{2}-J_{3}{ }^{-2 \nu /(1-2 v)}\right]-\mu(1-f)\left[\left(\lambda_{i}{ }^{-2}\right)^{-1}\right. \\
\left.-J_{3}{ }^{2 \nu /(1-2 \nu)}\right]
\end{array}
$$

(2) Strip-biaxial tension: For this case,

$$
\begin{aligned}
& \lambda_{1}=\lambda_{2} \lambda_{2}=1, \lambda_{3}=\lambda_{\text {th }} ; \sigma_{1}=\sigma_{\text {s-bi }}, \sigma_{2}=\sigma_{\text {lat }}, \sigma_{3}=0 \\
& J_{3}=\lambda \lambda_{\text {th }} \\
& \frac{\lambda \sigma_{s-\mathrm{bi}}}{\lambda^{2}-\lambda_{\mathrm{th}}{ }^{2}}=\mu\left[f+\frac{1-f}{\lambda^{2} \lambda_{\mathrm{th}}{ }^{2}}\right] \\
& f \mathrm{Yth}^{2}-J_{3}{ }^{-2 \nu /(1-2 \nu)}=(1-f)\left[\left(\lambda_{\mathrm{tl}}{ }^{-2}\right)^{-1}-J_{3}^{2 \nu /(1-2 \nu)}\right]
\end{aligned}
$$


Note that (54) is obtained from (40) by setting $i=1, j=3$; while (55) is obtained from (51) by setting $i=3$. An additional check on the theory would be provided by measurement of $\sigma_{\text {lat }}$; this, however, is difficult, and so, the equation corresponding to $i=2$, is not used.

Solution of (5j) leads to the simple result:

$$
\lambda_{t h}=J_{3}^{-\nu /(1-2 \nu)}
$$

or

$$
\lambda_{\mathrm{th}}=\lambda^{-v /(1-\nu)}
$$

Note that these expressions linearize to exactly the result that is given by infinitesimal theory, thus justifying the interpretation of the parameter $\nu$ as a large strain Poisson's ratio.

3. Homogeneous Biaxial Tension: For this case,

$$
\begin{gathered}
\lambda_{1}=\lambda_{2}=\lambda, \lambda_{3}=\lambda_{\text {th }} ; \sigma_{1}=\sigma_{2}=\sigma_{\mathrm{h}-\mathrm{bi},} \sigma_{3}=0 \\
J_{3}=\lambda^{2} \lambda_{\text {th }}
\end{gathered}
$$

The constitutive relations are identical with (54) and (55). The solution of (55) in this case, however, leads again to (56), but thence to:

$$
\lambda_{\mathrm{tl}_{2}}=\lambda^{-2 \nu_{i}(1-2 \nu)}
$$

Equation (60) differs from (57) only by the presence of the factor 2 in the numerator of the exponent which arises from the equal deformations imposed on two coordinates.

\section{Experimental Adductions}

\section{A. Test Setup}

In order to test the hypotheses:

(a) that $W_{1}$ and $W_{2}$ or $\{\mu, f\}$ are constant for some rubbers

(b) that dilatation may be expressed by (49) with $\nu$ a constant parameter, tensile tests were rum on two polyurethane rubbers:*

(1) a continum formulation containing $12 \times 10^{-5}$ moles of effective elastic chain per cc, as determined by swelling;

(2) a foamed binder prepared by incorporating and then leaching out $47 \mathrm{vol} . \%$ of salt from the above formulation.

* Courtesy of K. W. Bills, Aerojet General Corporation, Azusa, California. 
The continuum formulation was evaluated only in simple tension merely to demonstrate the mechanies by which $W_{1}$ and $W_{2}$ can be calculated for a polyurethane rubber, hitherto unreported in the literature. The foam rubber was tested in uniaxial, strip biaxial, and homogeneous biaxial tension. All tests were conducted on an Instron machine.

Figures 1 and 2 show the technique used to evaluate simple tension.

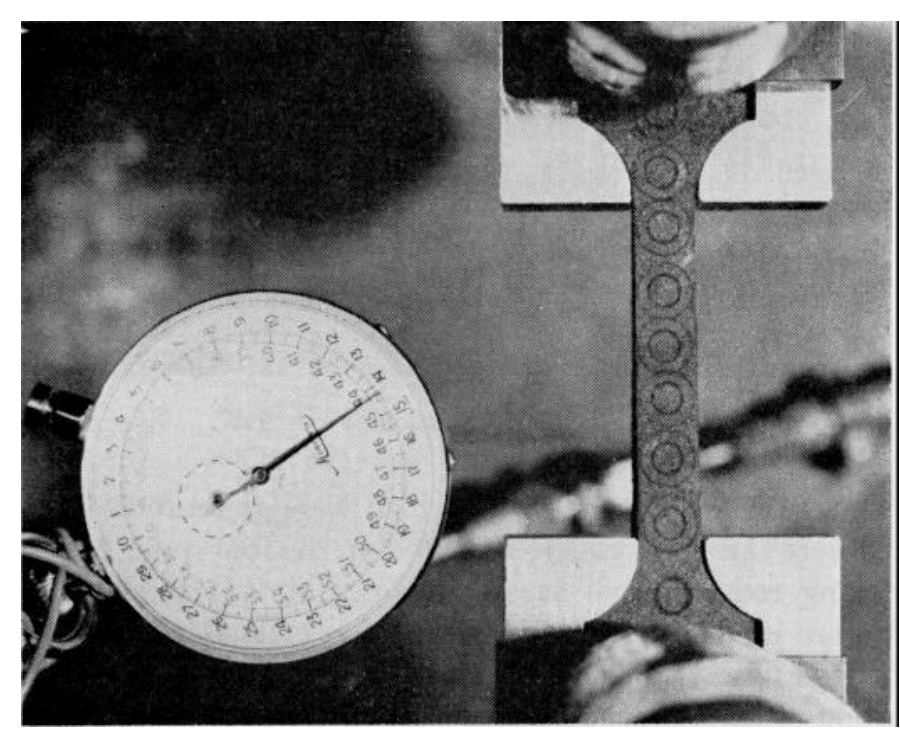

Fig. 1. Uniaxial tenaion at $\lambda=1$.

A grid of circles was inked on the gage section of dog-bone specimens with the use of Calco G L Fast dye. Excellent edge definition is obtained with the use of this ink, particularly if a thin film of aluminum paint is sprayed on first to provide optical contrast. Negative photographs of the circles and deformed ellipses were read under an optical comparator to a preeision of less than $1 \%$ for strains in excess of $20 \%$.

The strip-biaxial deformation was engendered in wide rectangular thin sheets $\left(7^{\prime \prime} \times 1^{\prime \prime} \times{ }^{3} / 1^{\prime \prime}\right)$ by glueing two pairs of rigid metal plates to both of the long edges, and thence pulling normal to these edges. The homogeneous-biaxial field was engendered in square 


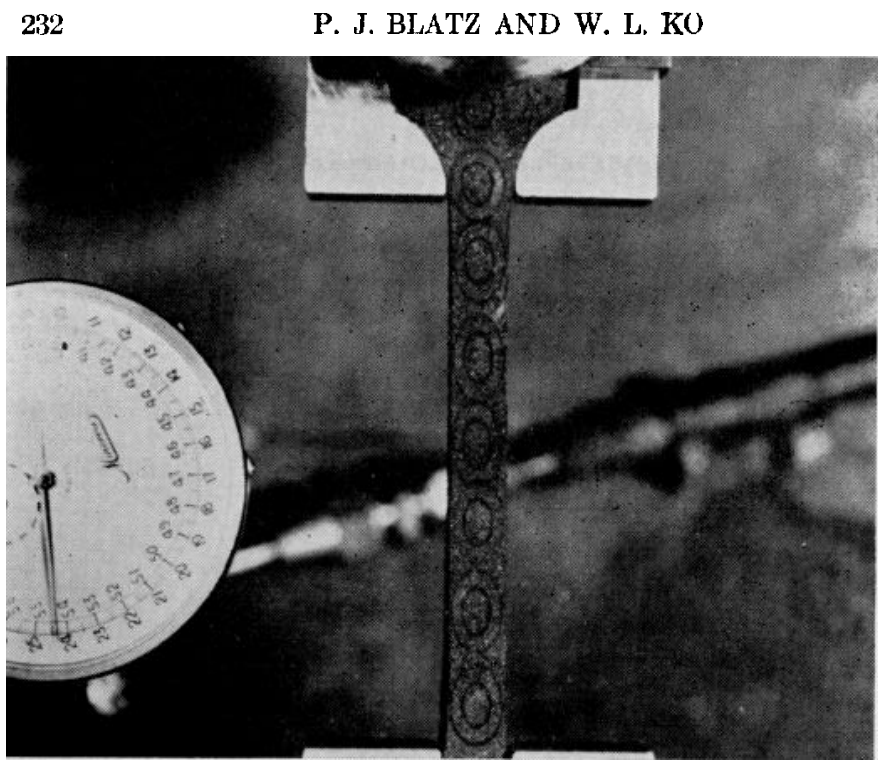

Fig. 2. Uniaxial tension at $\lambda=1.57$.

thin sheets $\left(3^{\prime \prime} \times 3^{\prime \prime} \times{ }^{3 / 16^{\prime \prime}}\right)$ to which were glued retractors which in turn were bent over rollers mounted on the four outer edges of two boomerang rods. In all cases, only equilibrium measurements at $75^{\circ} \mathrm{F}$. were made, so that the application of load was continuously interrupted until all friction effects were adjusted to zero until slight relaxation of the rubber had died out. Figures 3 and 4 show the experimental setup for the two biaxial fields and the technique used for measuring thickness with a dual traversible micrometer. Figures :5 and 6 show the strip before and after deformation. Figures 7 and 8 show the quadrangle before and after deformation.

\section{B. Data Reduction}

1. Continuum Rubbers: Figure 9 shows the equilibrium uniaxial load-deformation curve obtained on the continuum rubber. Figure 10 plots the dimensional changes $\left(\lambda_{\text {lat }}-\lambda\right)$. From these data the ratio (43) was plotted in Figure 11. Yote the excellent straight line, the slope of which is zero, indicating that $W_{2}=0, f=1$; the intercept

* The initially low point is presumed to be calsed by seating of the sample in the grips. 


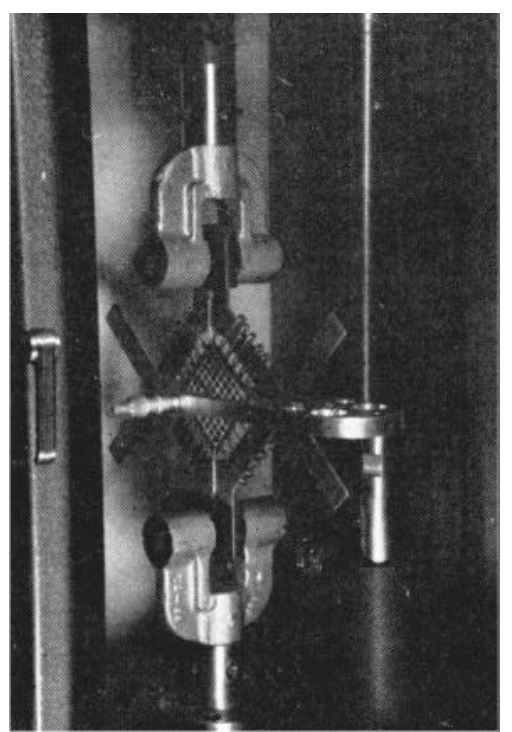

Fig. 3. Homogeneous-biaxial tension.

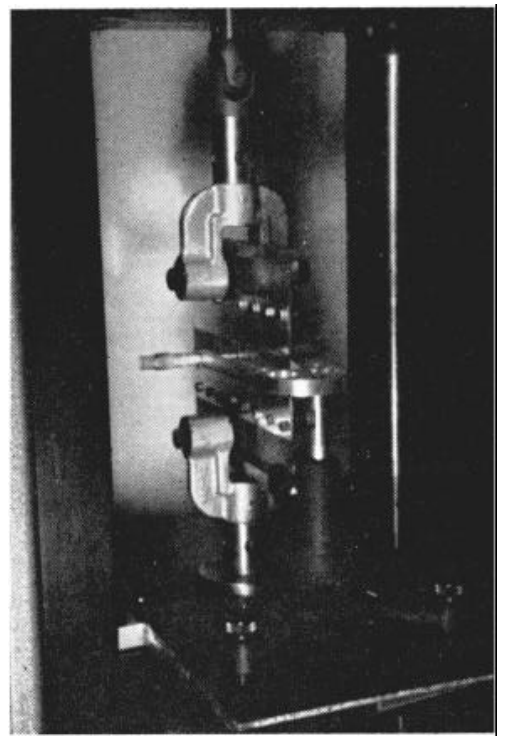

Fig. 4. Strip-biaxial tension. 

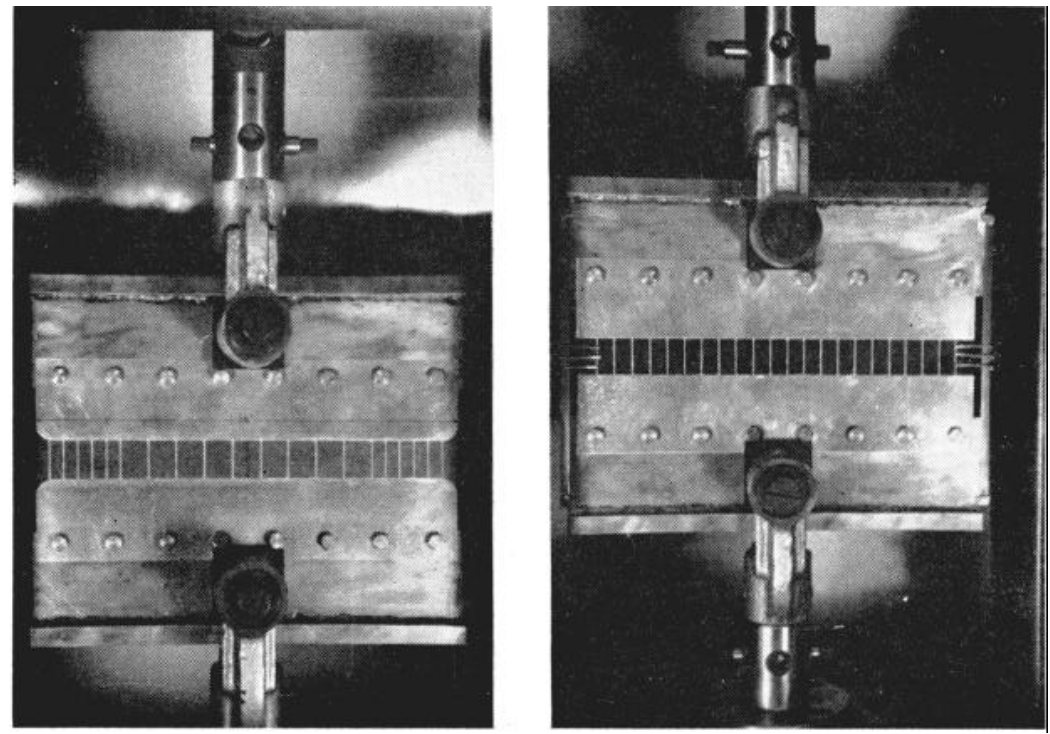

Fig. 5. Two types of strip-biaxial tension at $\lambda=\mathbf{1}$.

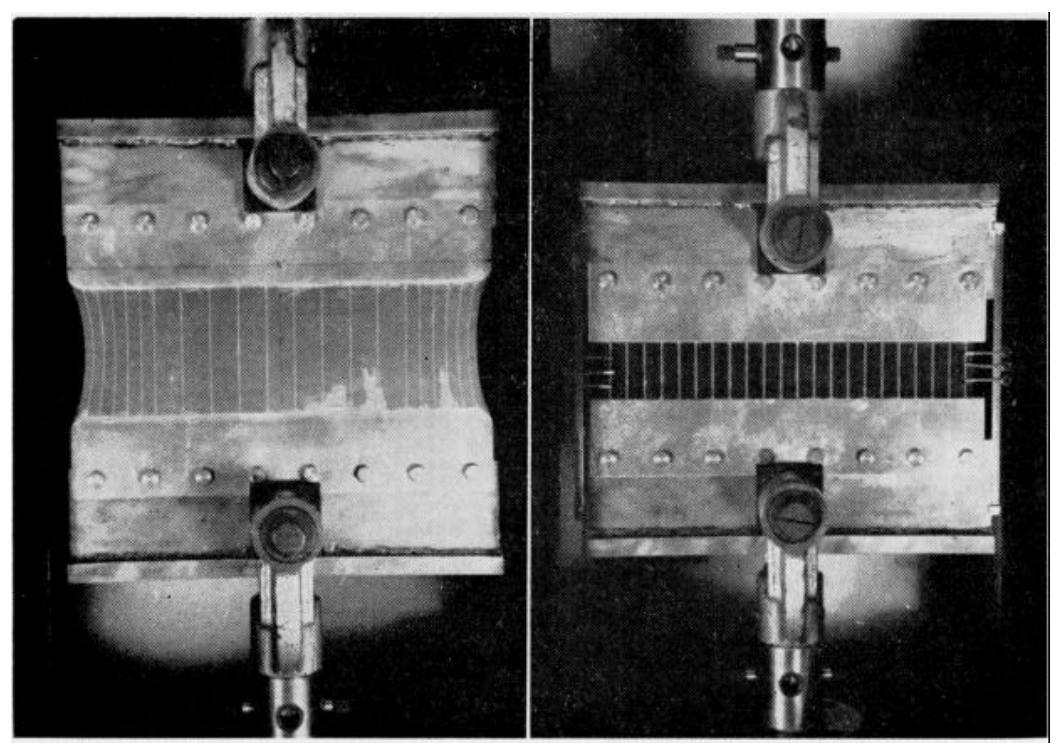

Fig. 6. Two types of strip-biaxial tension. Left: $\lambda=3.5$. Right: $\lambda=1.6$ 


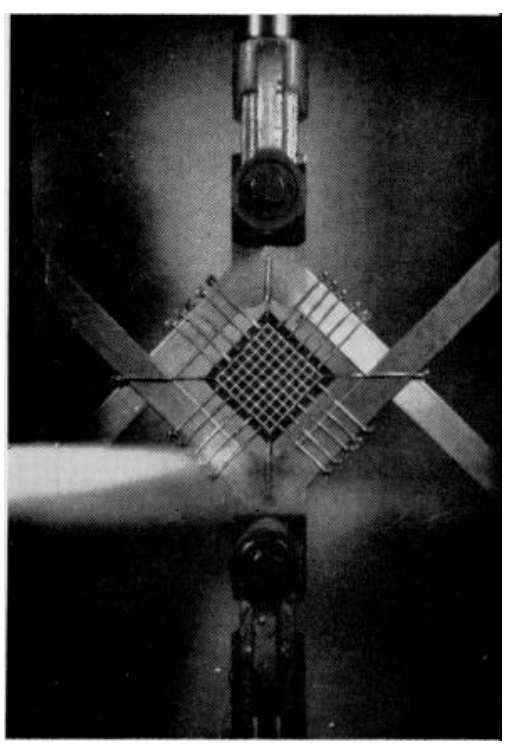

Fig. 7 . Homogeneoug-biaxial tension at $\lambda=1$.

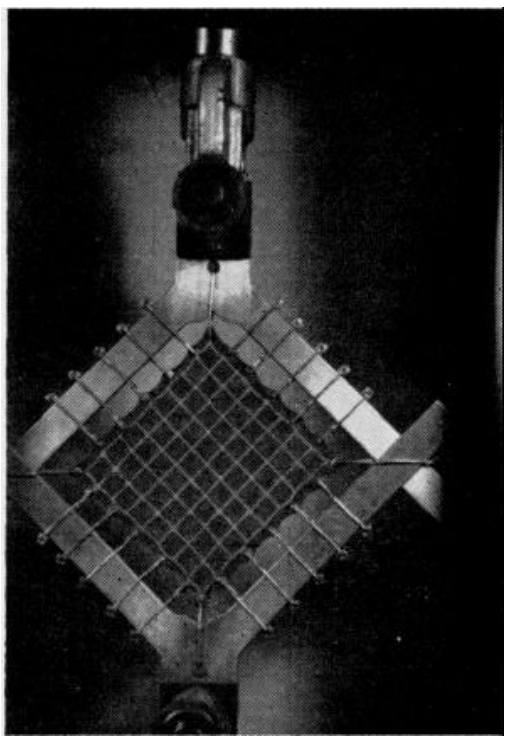

I.ig. ‥ Homogeneous-biaxial tension at $\lambda=2.33$. 


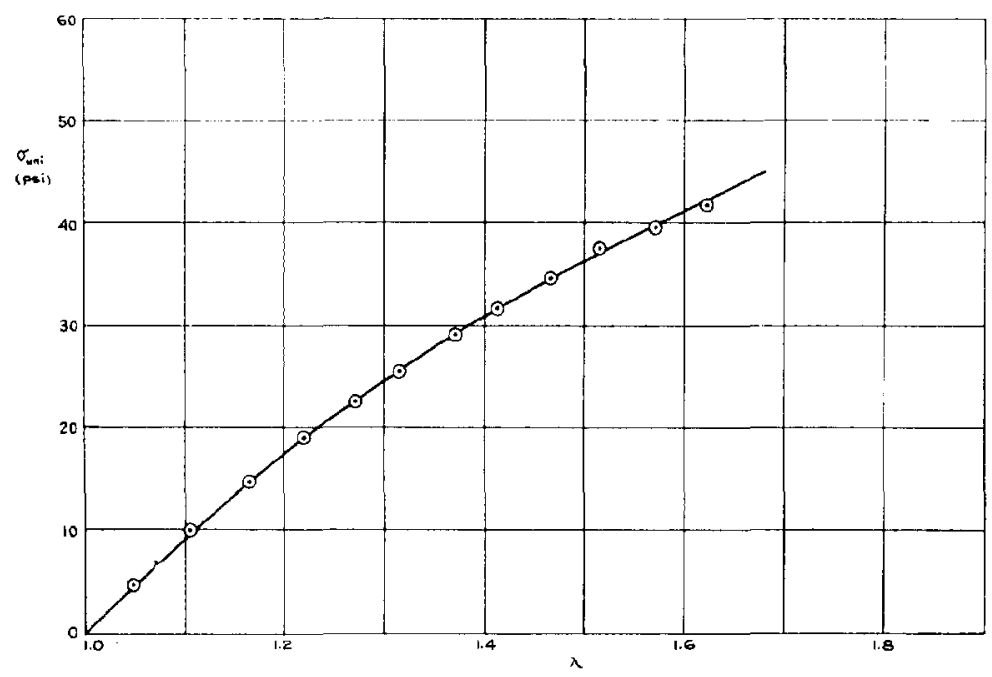

Fig. 9. Uniaxial stress vs, extension ratio (polyurethane rubber).

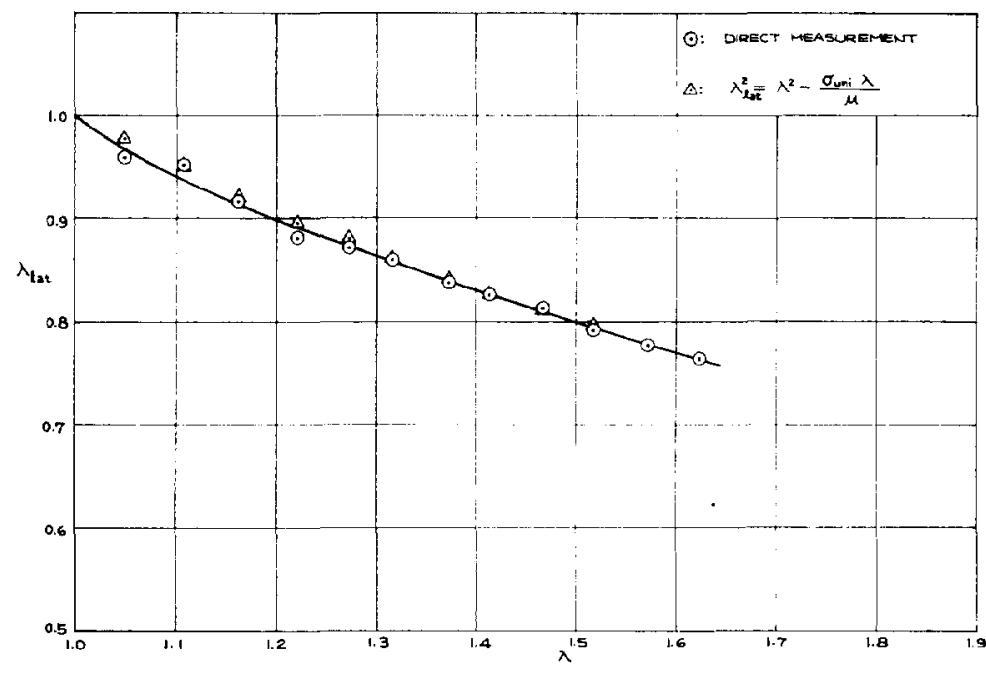

Fig. 10. Uniaxial lateral contraction ratio va. longitudinal extension ratio (polyurethane rubber). 


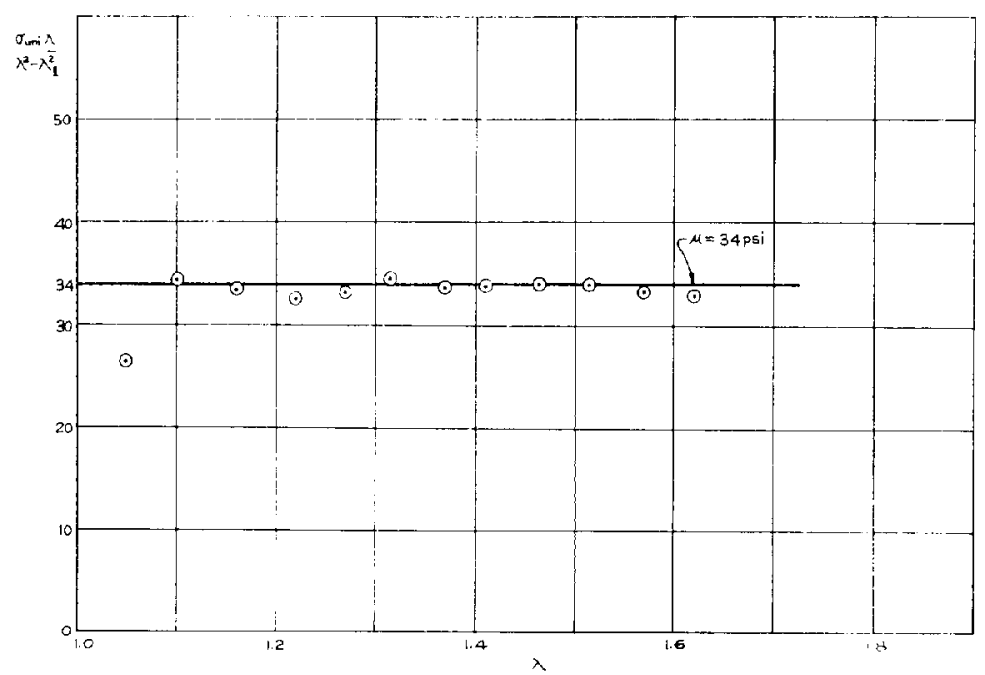

Fig. 11. Rectification of uniaxial data (polyurethane rubber).

of this line determines the shear modulus, $\mu=34$ p.s.i. Although $W_{2}$ is indeed zero for this polyurethane rubber, and all other polyurethane formulations investigated in this laboratory, this does not mean that $W_{2}$ is zero for all rubbers. ${ }^{7}$ Nor is this evidence in disagreement with any hitherto reported evidence. There is nothing in continuum mechanies that dictates the values of $\left\{W_{1} W_{2}\right\}$ other than that the leading terms of expansion in terms of small strain invariants must satisfy (24), (25), and (32).

Before passing on to a discussion of foam rubbers, it is useful to consider the interpretation of available compression data. This provides information about the nature of $W_{3}$, which information cannot be obtained from uniaxial or biaxial tests because of the low stresses involved and the high value of the exponent $(1-2 \nu)^{-1}$. Murnaghan $^{8}$ has fitted such data for the atomic alkali metals by a function of the form:

$$
P=\frac{K}{k}\left(J_{3}^{-k}-1\right)
$$

and found $k$ to be of the order of 4 . Remembering that $J_{3}$ is a measure of the cube of the change in interatomic distance, Murnaghan's 
observation suggests an inverse twelfth power dependence for what resembles a repulsive term in an interatomic potential function. ${ }^{9}$ Similar functional behavior is shown for butyl tread rubber ${ }^{10}$ in Figure 12 where Bridgman's data up to 700,000 p.s.i. are plotted.

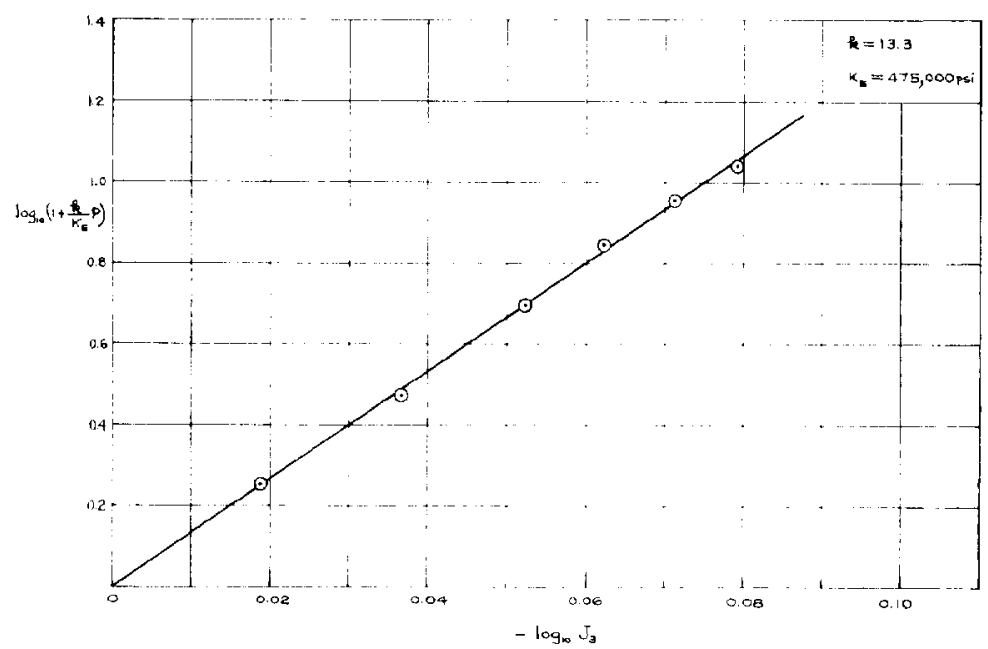

Fig. 12. Rectification of hydrostatic compression data-butyl tread rubber.

The excellent straight line is characterized by values of $k=13.3$ and $K=475,000$. This value of the bulk modulus compares favorably with data ${ }^{11}$ on polyisobutylene. The constitutive relation corresponding to (61) with $W_{2}=0$ and $W_{1}=\mu / 2$ is given by:

$$
\sigma_{i} \lambda_{i}=\mu\left(\lambda_{i}^{2}-J_{3}^{2 / 3}\right)+\frac{K J_{3}\left(1-J_{3}^{-k}\right)}{k}
$$

Equation (62) can be modified very easily to a form which reduces to (51), with $f=1$ :

$$
\sigma_{i} \lambda_{i}=\mu\left(\lambda_{1}^{2}-J_{3}^{2 / 3}\right)+\frac{K J_{3}^{m}}{k-l}\left(J_{3}^{-l}-J_{3}^{-k}\right)
$$

This becomes identical with (51) after choosing:

$$
k=\frac{5+2 \nu}{6(1-2 \nu)}
$$




$$
\begin{aligned}
& l=\frac{1}{6} \\
& m=\frac{5}{6}
\end{aligned}
$$

The term in $J_{3}^{-l}$ represents the attraction component of the interchain potential and is so small with respect to the term in $J_{3}^{-k}$ at high stresses that it is not detectable in the range of the data plotted in Figure 12. We suggest (63) as a new equation of state for continuum rubbers $(f=1)$ valid up to very high compressive stresses. In order to evaluate " $l$ " and " $m$ " precisely, data must be procured in hydrostatic tension, which is not easy to do. Efforts are being made in this direction.

The reason why the value of $k=13.3$ is larger than that which Murnaghan found for monatomic molecules probably lies in the fact that the small monomeric units are tied together in long polymer chains. This in some way affects the value of $k$ and is the basis for an interesting molecular statistical investigation. From (64), it develops that the finite strain value of Poisson's ratio is 0.463 , so that rubber is not nearly as incompressible as the linear theory would indicate.

2. Foam Rubbers: Figure 13 shows the uniaxial load-deformation curve averaged through four sets of data obtained on the foam rubber. Two of the samples fractured at $140 \%$ strain. These are the values beyond which the sample is caused to snap by a slight increase in load. Thus these so-called ultimate values say nothing about the details of the fracture mechanics, but merely bound the strain level (within $5 \%$ ) within which fracture initiates in a given sample. Larger (than $5 \%$ ) sample-to-sample variations will depend on the presence of local defects in the sample and local strain concentrations set up around these defects by virture of the particular method of gripping and will also depend on the dimensions of the sample. A so-called isotropic failure criterion is obtained when the standard deviation of the measured ultimate values becomes independent of sample dimensions in the limit of large dimensions. ${ }^{12} \quad$ Studies of size effects are being pursued. Once obtained, the isotropic failure criterion is representative of the fracture of a large sample with randomly distributed local defectsthe effects of which are averaged out in testing a large number of samples. 


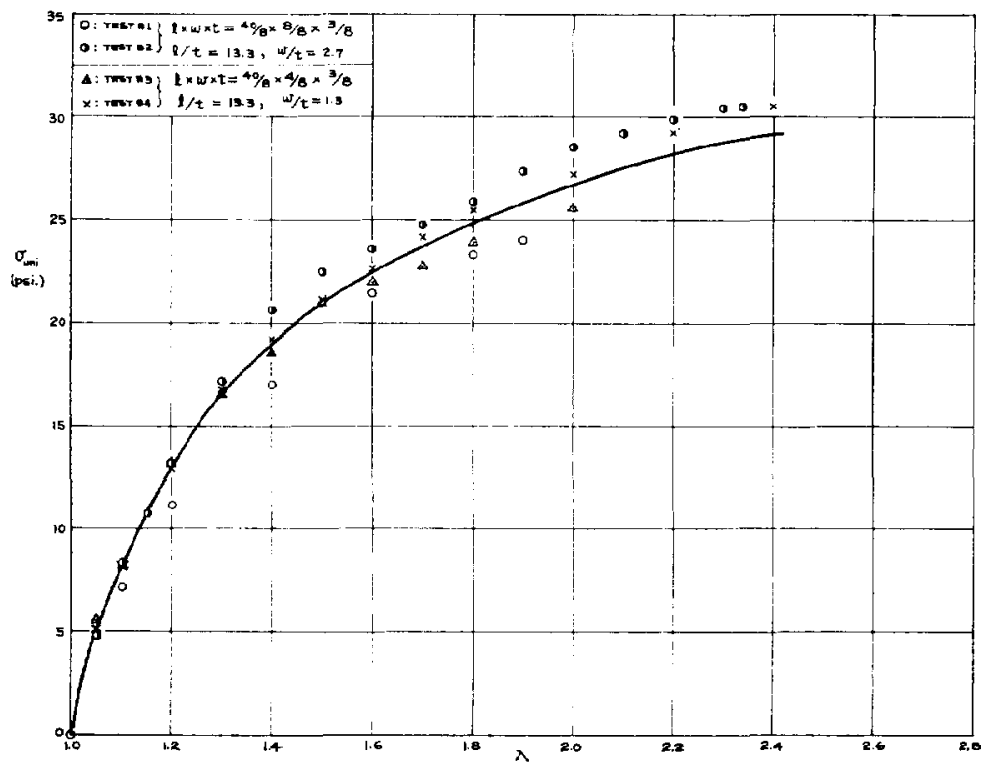

Fig. 13. Uniaxial stress vs. longitudinal extension ratio (polyurethane foam).

This criterion does not give a true picture of fracture initiation. In order to obtain such, one would have to prepare defect-f ree samples, and presumably have to work in the region of relatively small dimensions. This is extremely difficult to do, as evidenced by the analogy with studies on single crystals in metals. An alternative procedure is to introduce a well defined crack or notch and study the growth of this defect. Such studies are also under way at GALCIT.

The dimensional ehanges associated with the uniaxial test are shown in Figure 14. The curve of Figure 13 is rectified when plotted in Figure 15 in the manner suggested by (43). It is observed that, for the foam as opposed to the continuum rubber, $W_{1}$ is small, approximately zero, whereas $W_{2}$ is large and positive, so that, figuratively speaking, all the shear behavior arises from the second MooneyRivlin-type constant. When the data of Figure 14 are replotted in the form suggested by (46), namely $\ln J_{3}$ vs. $\ln \lambda$, in Figure 16 , there results a straight line with slope $1 / 2$, giving Poisson's ratio a value of $1 / 4$. This value is used to predict theoretical slopes for the data obtained in the other two stress fields. 


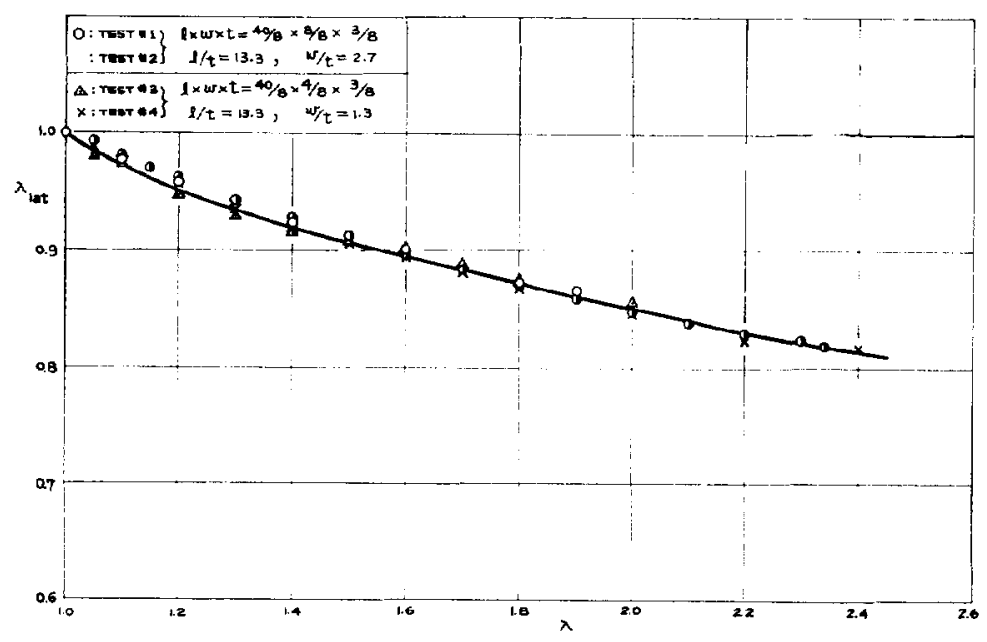

Fig. 14. Uniaxial lateral contraction ratio vs. longitudinal extension ratio (polyurethane foam).

Figure 17 shows the strip-biaxial load-deformation curve and Figure 18 the associated dimensional changes. After rectification of the load-deformation curve (Fig. 19), it is again observed that $W_{1} \approx 0$. Likewise, Figure 20 shows the excellent agreement evinced between the $\log -\log$ dilatation data and the theoretical line based on $\nu=1 / 4$. Figures 21-24 show the same respective data and results for homogeneous biaxial data.

The results of these studies are summarized in Table $I$.

It follows that the general stress-deformation behavior of a 47 vol. $\%$ foamed polyurethane rubber is completely contained within

TABLE I

Results of Studies

\begin{tabular}{lccc}
\hline \multicolumn{1}{c}{ Type test } & $\begin{array}{c}\text { Shear } \\
\text { modulus(p.s.i.) }\end{array}$ & $f$ & $\begin{array}{c}\text { Poisson's } \\
\text { ratio }\end{array}$ \\
\hline Simple tension & 38 & 0.13 & $1 / 4$ \\
Strip-biaxial tension & 29 & 0.07 & $1 / 4$ \\
Homogeneous-biaxial tension & 27 & -0.19 & $1 / 4$ \\
\multicolumn{1}{c}{ Average value } & - & $-1 / 4$ & $1 / 4$ \\
\hline
\end{tabular}




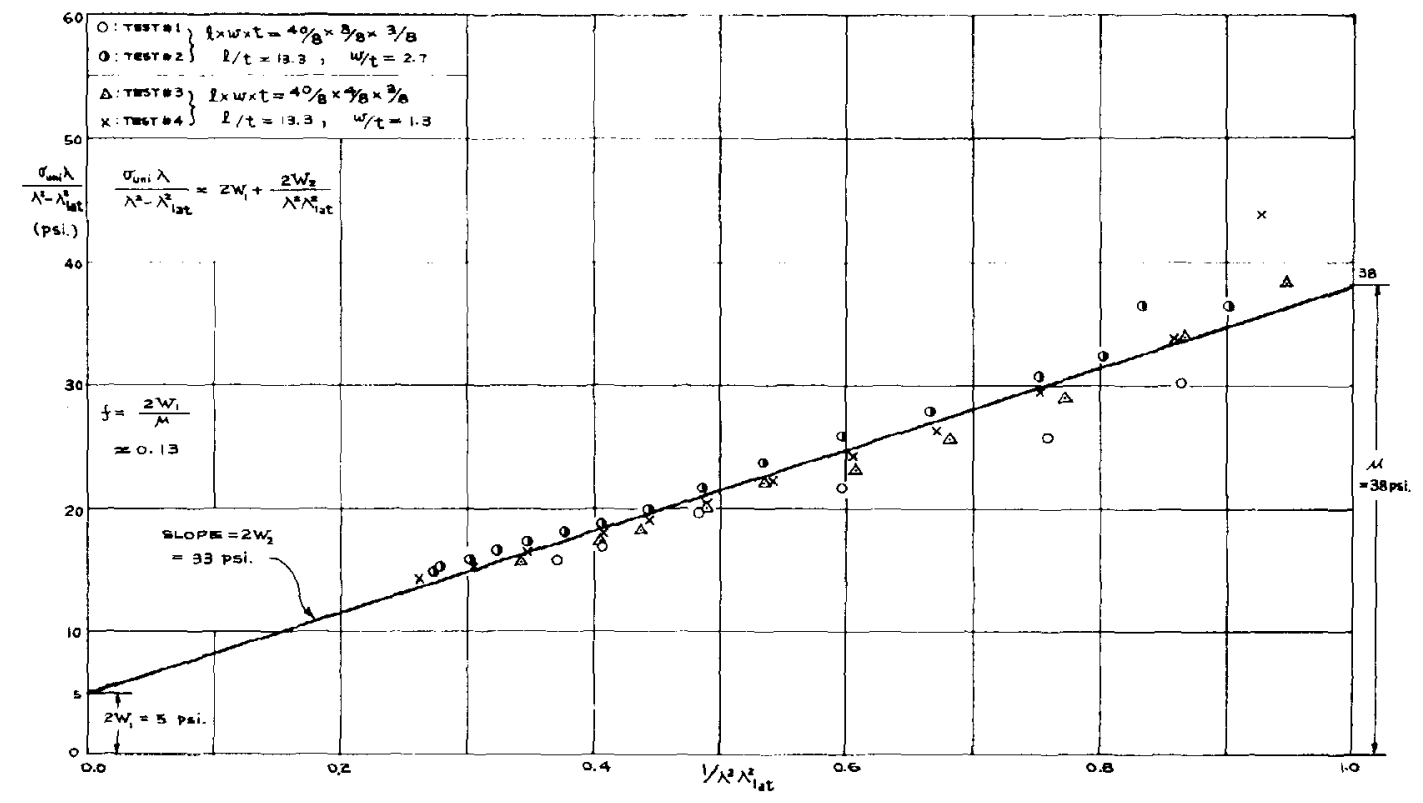

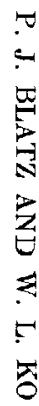

Fig. 15. Vualuation of $W_{1}$ and $W_{2}$ from rectified uniaxial data (polyurethane foum). 


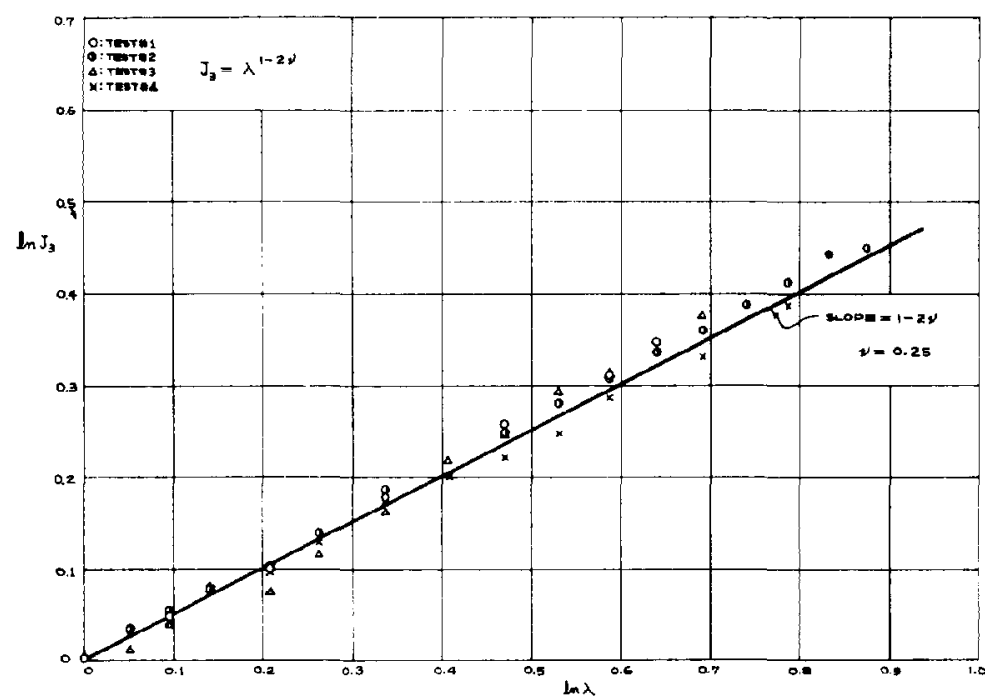

Fig. 16. Dependence of uniaxial dilatation on longitudinal extension ratio (polyurethane foam).

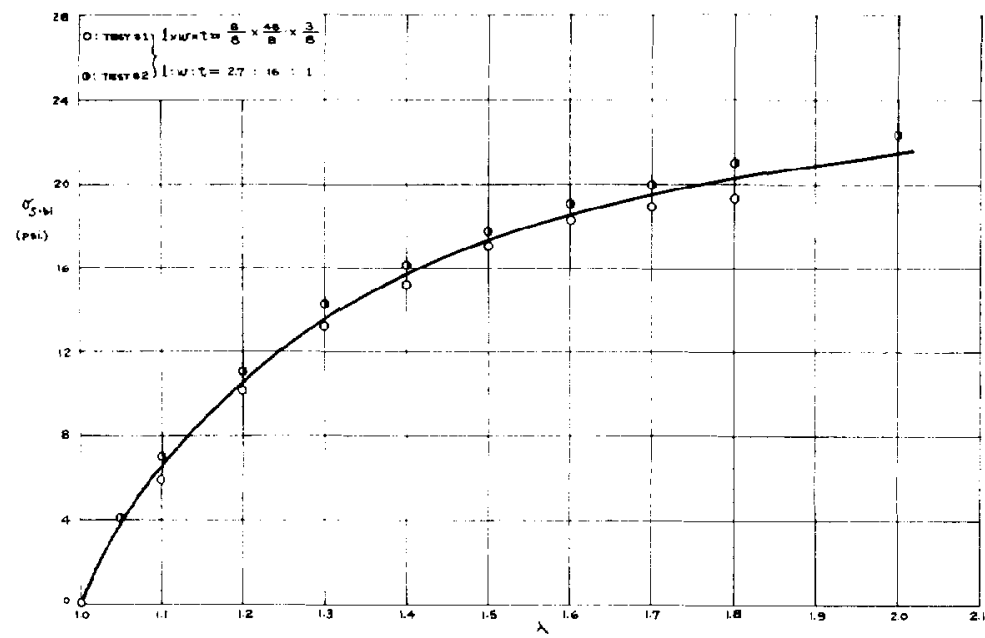

Fig. 17. Strip-biaxial stress vs. longitudinal extension ratio (polyurethane foam). 


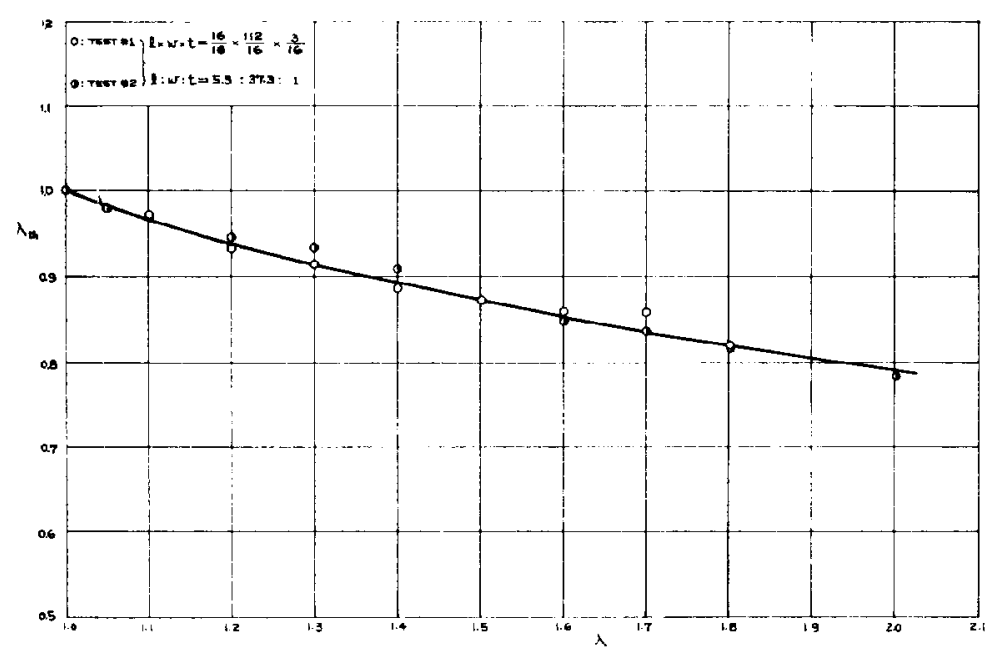

Fig. 18. Strip-biaxial thickness contraction ratio vs. longitudinal extension ratio (polyurethane foam).

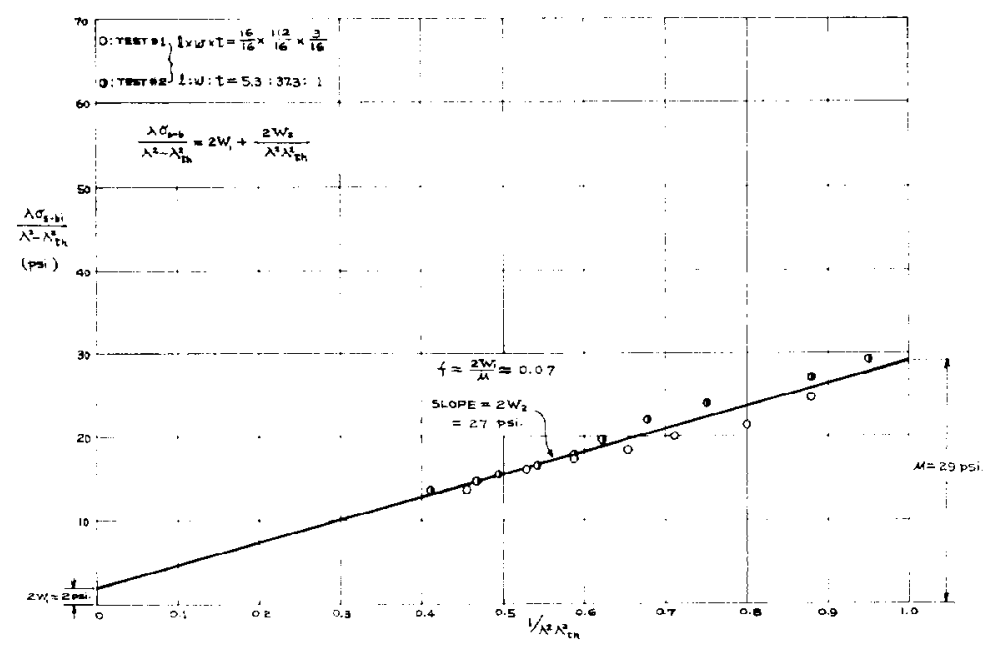

Fig. 19. Evaluation of $W_{1}$ and $W_{2}$ from rectified strip-biaxial data (polyurethane foam). 


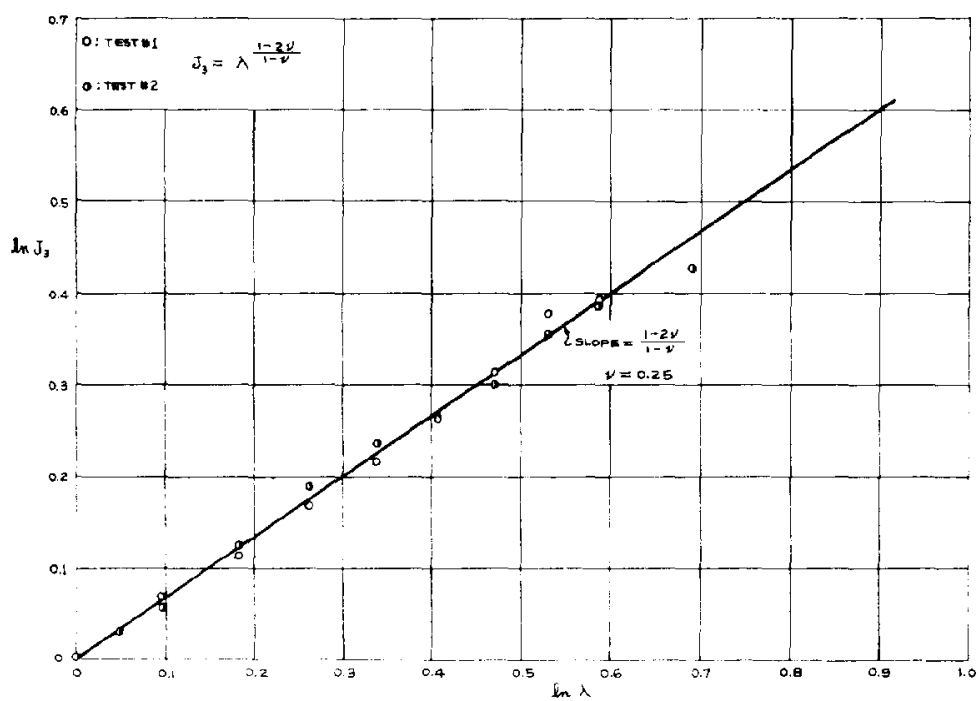

Fig. 20. Dependence of strip-biaxial dilatation on longitudinal extension ratio (polyurethane foam).

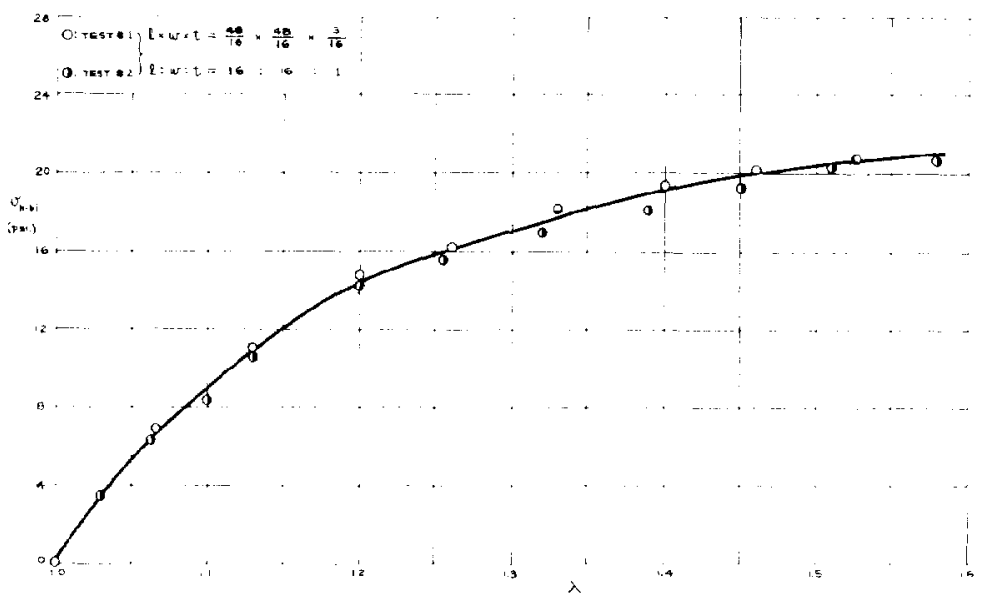

Fig. 21. Honogeneous-biaxial stress vs. longitudinal extension ratio (polyurethane foam). 


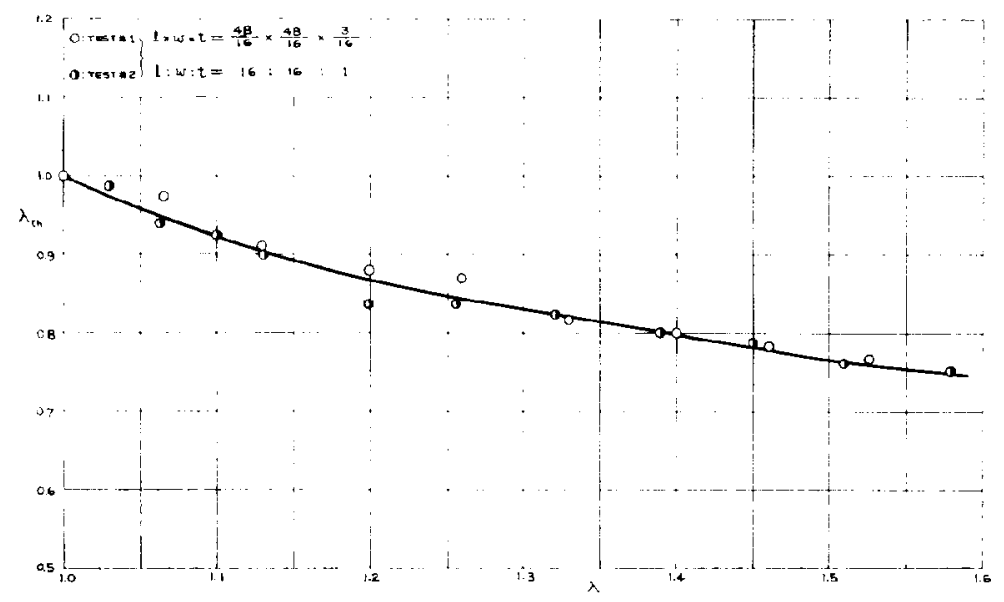

Fig. 22. Homogeneous-biaxial thickness contraction ratio vs. longitudinal extension ratio (polyurethane foam).

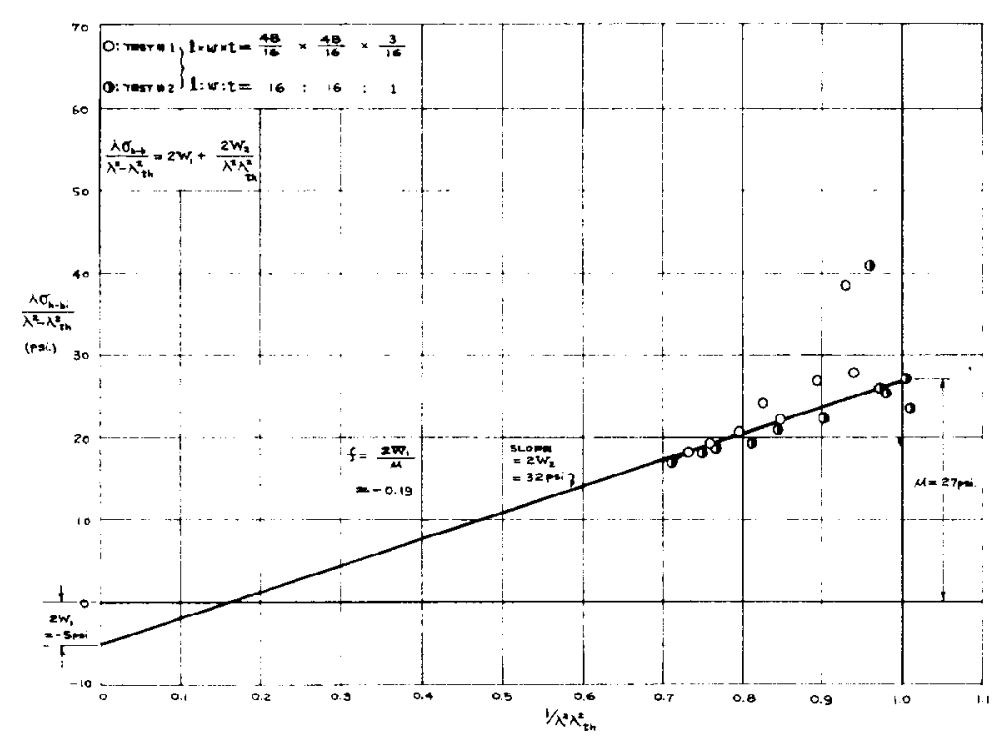

Fig. 23. livaluation of $W_{1}$ and $W_{2}$ from rectified homogeneous-biaxial data (polyurethane foam). 


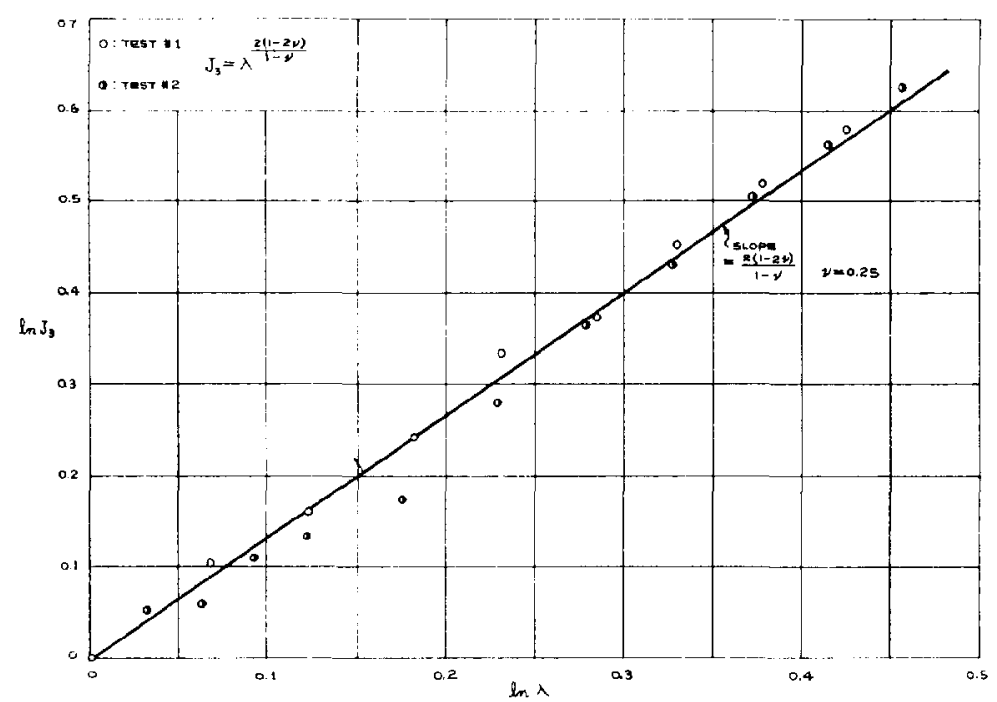

Fig. 24. Dependence of homogeneous-biaxial dilatation on longitudinal extension ratio (polyurethane foam).

the strain energy function (50), with the experimentally assigned values $\{f=0, \nu=1 / 4\}$, for which:

$$
\begin{gathered}
W=\mu / 2\left(J_{2}+2 J_{3}-5\right) \\
\hat{\sigma}_{i} J_{3}=\sigma_{i} \lambda_{i}=\mu\left[J_{3}-\left(\lambda_{i}{ }^{2}\right)^{-1}\right]
\end{gathered}
$$

\section{The Failure Criterion}

Equation (68) shows that the maximum achievable value of true stress in an infinitely extensible foam rubber of the type previously defined and subjected to any arbitrary tensile stress field, is its shear modulus:

$$
\bar{\sigma}_{i}=\mu\left[1-\left(\lambda_{i}{ }^{2} J_{3}\right)^{-1}\right] \leqslant \mu
$$

This immediately sets an upper bound to the stresses expected in a deformed foam. On the other hand, the minimum value obtained in compression may theoretically approach infinity, or practically, some value of the order of millions of atmospheres, beyond which atomic neighbors proceed to fuse into neutron matter. Somewhere en route 
to this "dwarf" state, there may, of course, be failure associated with buckling of the foam structure, tearing of the polymer chains, and crushing of the electron shells. Just where these types of failure occur is of no present interest, and so we shall define ${ }^{13}$ the supremum of all failure surfaces in principal stress space as a cube, three faces of which intersect the positive $\{i, j, k\}$ axes at each of three points a distance " $u$ " from the coordinate origin, and which intersect mutually at an apex which lies on the positive ray of the hydrostatic vector at a distance $\mu \sqrt{3}$ from the coordinate origin. The other three faces of this cube will intersect the negative $\{i, j, k\}$ axes at each of three points whose distance from the origin is unspecified, but which may well be in excess of a million p.s.i. The actual failure surface for any foam which is represented by a constitutive law of type (69) lies within this cube.

Equation (69) is nonlinear and multi-valued in the displacements, so that to a given surface in principal stress space is associated a plurality of surfaces, or perhaps a whole region, of principal deformation space. Likewise, to a given surface in $\lambda$-space there is associated a plurality of surfaces in principal stress space. This lack of uniqueness is highlighted by inverting (69) to yield:

$$
\lambda_{i}^{2}=\frac{m_{i}}{\left(m_{i} m_{j} m_{k}\right)^{1 / 5}}
$$

where

$$
m_{i}=\left(1-\frac{\bar{\sigma}_{i}}{\mu}\right)^{-1}
$$

It follows that:

$$
\begin{gathered}
J_{3^{5}}=\left(m_{i} m_{j} m_{k}\right)=M_{3} \\
J_{1} J_{3}=\Sigma m_{i}=M_{1} \\
J_{2} J_{3}=\Sigma m_{i}^{-1}=M_{2}
\end{gathered}
$$

Thus, the strain-energy function can also be cast as a function of the reduced-stress invariants, based on the reduced stresses given by (71). And the failure surface may be depicted in $\bar{\sigma}_{i}$-space, $\lambda_{i}$-space, $J_{i}$-space, or $M_{i}$-space. Which of these spaces is used is at present a matter of convenience. It may turn out that the topological 
features of a failure surface are best demonstrated in one of these four. This is a fruitful area for investigation.

Returning to $\tilde{\sigma}_{i}$-space it is observed that simple geometrical interpretations can be ascribed to the various criteria that have been proposed to explain failure. For example, the mean-stress resultant $\sqrt{\Sigma \bar{\sigma}_{i}^{2}}$ depicts a sphere, centered at the coordinate origin. The mean-stress deviator $\sqrt{\sum_{i \neq j}\left(\bar{\sigma}_{i}-\bar{\sigma}_{j}\right)^{2}}$ depicts a cylinder coaxial with the hydrostatic vector, the radius of which equals $\sqrt{2}$ times the maximum shear stress the material can withstand. The hydrostatic stress, or first stress invariant depicts a plane normal to the hydrostatic vector which it intersects at a point removed a distance from the origin equal to $\sqrt{3}$ times the maximum hydrostatic tension which the material can withstand. This plane caps the cylinder previously alluded to. Finally the second stress invariant depicts a dish-shaped triangular hyperboloid, ef. Figure 25.

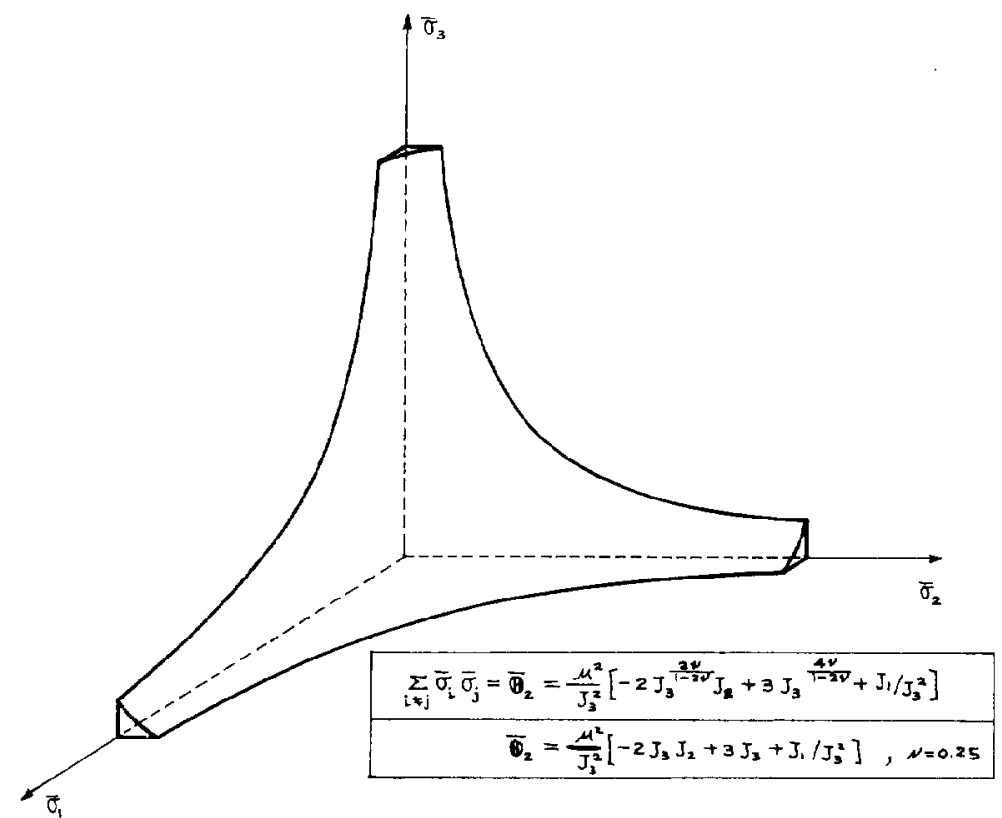

Fig. 25. Failure safe surface based on second true stress invariant in normal stress space. 
For each of these criteria, there is an associated geometry in J-spare defined by:

$$
\begin{gathered}
\sqrt{\Sigma \bar{\sigma}_{i}^{2}}=\mu \sqrt{\left(3-2 \frac{J_{z}}{J_{3}}+\frac{J_{2}^{2}}{J_{3}^{2}}-\frac{2 J_{1}}{J_{3}^{4}}\right)} \\
\sqrt{\sum_{i \neq j}\left(\bar{\sigma}_{i}-\bar{\sigma}_{j}\right)^{2}}=\mu \sqrt{\left(2 \frac{J_{2}^{2}}{J_{3}^{2}}-6 \frac{J_{1}}{J_{3}{ }^{4}}\right)} \\
\Sigma_{\bar{\sigma}_{i}}=\mu\left(3-\frac{J_{2}}{J_{3}}\right) \\
\sqrt{\sum_{i \rtimes j} \bar{\sigma}_{i} \bar{\sigma}_{j}}=\mu \sqrt{3-2 \frac{J_{2}}{J_{3}}+\frac{J_{1}}{J_{3}}}
\end{gathered}
$$

In addition, one can depict surfaces which correspond to the mean resultant strain, the mean deviatoric st rain, and so on.

(f) all the surfaces investigated, the data plotted in Figure 26 based

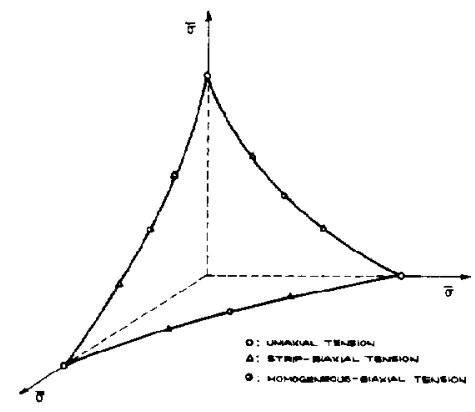

Fig. 26. Failure sate surface based on experimental data in normal stress space.

on the experimental values of the ultimate principal stresses (divided by shear modulus to eliminate sample-to-sample variation) seem to generate a surface most like that of Figure 25. Data are now being procured in stress fields close to hydrostatic tension in order to evaluate the depth of the disc.

\section{References}

1. Hooke, R., Lecturac de Potentia Restitutiva. London (1678).

2. Rivlin, R. S., Phil. Trans. Roy. Soc. London, A240, 459 (1948).

3. Reiner, M., Am. J. Math., 70, 433 (1948). 
4. Truesdell, C., J. Ratl. Mech. and Anal., 1, 125 (1952).

5. Rivlin, R. S., Phil. Trans. Roy. Soc. London, A240, 491 (1948); A240, 509 (1948); A241, 379 (1949); Proc. Roy. Soc. (London), A195, 4633 (1949); Phit. Trans. Roy. Soc. London, A242, 173 (1950); A243, 251 (1951).

6. Truesdell, C., see ref. 4.

7. Flory, P, J., and R. Ciferri, J. A ppl. Phys., 30, 1498 (1959).

8. Murnaghan, F., Finite Deformation of an Elastic Solid, John Wiley and Sons, Ine., Now York, 1951.

9. Classtone, S., Theoretical Chemistry, D. Van Nostrand, New York, 1944, p. 430 .

10. Bridgman, P., Proc. Ame. Acad. Arts Sci., 76, 9 (1945).

11. Marvin, R.S., and R. Aldrichi, J. Appl. Phys., 25, 1213 (1954).

12. Irwin, G., "Fracture," in Handhuch der Physik, Vol. II, S. Flïgge, ed., Springer-Verlag, Berlin, 1955.

13. Blatz, P. J., The Yield Surface in Normal Stress or Normal Strain Space (GALCIT SM 60-14. California Institute of Technology). 19th Meeting Bulletin of JAXAF Physical Properties Panel, p. 165, September, 1960.

\section{Synopsis}

The notation of finite elastic theory is introduced. 'The mechanics of data reduction needed to determine the parameters of the theory are displayed. Experimental data, obtained in three different stress fields, are adduced for a foamed polyurethane rubber. A strain energy function is generated which correlates all the data to a high degree of accuracy. This new strain energy function is cast in terms of a constant $\nu$ (which has the same significance as Poisson's ratio in intinitesimal theory). A geometrical evaluation of the failure criterion is presented. 